\title{
MUAC-for-age more useful than absolute MUAC for nutritional surveillance in Somalia: results from nineteen cross-sectional surveys (2007-2016)
}

Estefania Custodio $^{1 *+} \mathbb{D}$, Rocio Martin-Cañavate ${ }^{1 \dagger}$, Federica Di Marcantonio ${ }^{1}$, Daniel Molla², Yusuf Abukar ${ }^{2}$ and Francois Kayitakire ${ }^{1}$

\begin{abstract}
Background: Somalia is affected by a civil war and a protracted humanitarian crisis for more than two decades. The international community has put in place nutrition surveillance systems to monitor the situation and inform decisions. However, the indicators commonly used to identify acute malnutrition, weight-for-height Z-score (WHZ) and mid upper arm circumference (MUAC), do not always converge in their estimations of acute malnutrition, creating challenges for decision making. Furthermore, the divergences are not consistent across livelihood populations within the country. We explored the MUAC-for-age Z-score (MUACAZ) as an alternative indicator in Somalia to minimize the discrepancy.
\end{abstract}

Methods: We analyzed data from nineteen cross-sectional surveys conducted in Somalia between 2007 and 2016. We compared the acute malnutrition prevalence estimates by each of the indicators and the degree of overlap in the individual diagnosis of acute malnutrition between the WHZ and the MUAC-based indicators. We performed multivariate regression analysis with sex, age and stunting as independent variables and acute malnutrition as the dependent outcome, defined by WHZ, MUAC or MUACAZ. We performed all the analysis in the population overall and in each of the livelihood populations separately.

Results: A total 255,623 measurements of children 6-59 months of age were analyzed. The overall prevalence of global acute malnutrition by MUACAZ (15.8\%) was similar to the one obtained using WHZ (16\%), whereas prevalence based on MUAC was much lower (7.8\%). These patterns of divergence were sustained throughout the nineteen surveys and the livelihoods studied, with only few exceptions. However, the proportion of overlap in the individual diagnosis of children as acutely malnourished was low between WHZ and absolute MUAC diagnosis (18.1\%) and also between WHZ and MUACAZ (28.3\%). Results show that age, sex and stunting status of the child affected the likelihood of being diagnosed as acutely malnourished to varying degrees, depending on the indicator used.

(Continued on next page)

\footnotetext{
* Correspondence: estefania.custodio@ec.europa.eu

${ }^{\dagger}$ Equal contributors

'European Commission, Joint Research Centre, Via E. Fermi, 2749, 21027,

Ispra, Varese, Italy

Full list of author information is available at the end of the article
} 
(Continued from previous page)

Conclusions: The MUAC-for-age (MUACZ) indicator yielded acute malnutrition prevalence estimates convergent with those obtained by WHZ indicator. However, the degree of overlap between these two indicators for individual diagnosis of acute malnutrition is low. Further studies of MUACAZ as an alternative indicator for nutrition surveillance are needed.

Keywords: Acute malnutrition, Weight-for-height, MUAC, MUAC-for-age, Severe acute malnutrition, Children, Somalia, Africa

\section{Background}

Somalia has been affected by a civil war since 1991, with the food security situation further aggravated by recurrent and prolonged droughts. Located in the Horn of Africa, the combination of conflict and natural disasters has eroded its livelihoods, caused structural food insecurity, population displacements and extreme poverty [1]. The country has experienced two famines in its recent history: 1992/1993 and 2011/2012 and it is currently one of the four countries (including South Sudan, Nigeria and Yemen) that are facing risk of famine in 2017. Within this context, the rates of acute malnutrition have persistently remained at 'critically' high levels over the last two decades [2]. As part of the efforts of the international community to improve the conditions in the country, the Somalia Food and Nutrition Security Analysis Unit (FSNAU) has been collecting data on anthropometric indicators throughout time to monitor the situation.

On-going surveillance of anthropometric indicators is crucial to detect the deterioration of the nutritional status of a population. It can provide information on trends, allowing for comparison overtime and against baseline years results, and it permits for geographical and contextual comparisons which can inform the prioritization of actions and the allocation of resources [3]. For this purpose, however, it is essential that the indicators used to monitor the situation yield comparable results.

In two recent reviews of nutrition surveillance systems in humanitarian settings and low income countries [3, 4], the advantages and challenges of the different data collection systems were discussed in terms of validity, reliability and institutional management, but little attention was given to the anthropometric measures being collected, which have been discussed elsewhere [5].

The recommended anthropometric indices and measures for acute malnutrition surveillance are weightfor-height/length Z-score (WHZ), absolute value of MidUpper Arm Circumference (MUAC) and MUAC-for-age Z-score (MUACAZ), with the cut-off points detailed in Box 1, being WHZ and MUAC the most widely used.

However, and although the absolute MUAC cut-off points were selected from statistical analysis to best match the WHZ cut-off points, so that the same prevalence of acute malnutrition would be found with each criterion [6], it has been shown that the prevalence obtained when using either indicator are not convergent and that the children identified for admission to programs with either indicator are not the same $[7,8]$.

For this reason, in nutrition surveillance systems aimed at identifying children with severe acute malnutrition (SAM) for admission in feeding programs, the World Health Organization (WHO) and United Nations International Children's Emergency Funds (UNICEF) recommend the use of $\mathrm{WHZ}<-3$ and also the use of absolute MUAC below $115 \mathrm{~mm}$, as the latter indicator presents operational advantages in terms of time and cost of data collection, and is also believed to identify the children at higher risk of death [9]. Although the discussion regarding which of these two indicators is more correlated with mortality is still on-going $[7,10]$, the use of absolute MUAC in combination with or in replacement of WHZ has been spread for program screening by field organizations, as a practical tool to screen vulnerable children in need for treatment [11].

However, if the aim of the surveillance is to assess the severity of the situation in the population and the targeting of humanitarian actions, WHO has recommended the use of WHZ or the use of MUAC adjusted by age and sex, that is the MUACAZ, but not the use of absolute MUAC [12]. The main reason is that WHZ and MUACAZ take into account the sex and the age or the height of the child, whereas absolute MUAC is strongly age- and sex- dependent, thus malnutrition estimates are conditioned to the age and sex structure of the study population when this latter indicator is used [13]. However, as MUACAZ requires accurate age data collection, which may be a challenge in certain humanitarian contexts, the preferred indicator has been the WHZ, and it is the only indicator of acute malnutrition for which there are prevalence thresholds to support the public health interpretation of results at global level [12].

Nonetheless, in recent times, the use of the MUAC measurement (or absolute MUAC) below $125 \mathrm{~mm}$ has been advocated as a proxy for global acute malnutrition for population estimates, grounded on the operational advantages of the indicator described before [5]. This is 
creating major confusion amongst policy and decision makers, as the discrepancies in the acute malnutrition estimates when using the WHZ index or the absolute MUAC measurement are not consistent across populations [11]. This is highly problematic in terms of geographical and temporal comparisons of the nutrition situation across populations, even at the region or subregion level within a country.

The reasons for these discrepancies are not fully understood, but an important part of this variation must be linked to the age and sex dependency of the absolute MUAC measurement [8]. That is why MUACAZ was suggested as a more useful indicator of nutritional status for population estimates than absolute MUAC [14]. Moreover, the MUACAZ has been shown to be a sensible indicator to detect changes in the nutritional status of populations [15]. Therefore, and as it retains most of the operational advantages of the MUAC measurement, it may be an alternative indicator when the weight and height measurements are difficult to obtain. Nevertheless, little is known of how the acute malnutrition estimates using MUACAZ relate to the ones obtained using WHZ, as the MUACAZ indicator has been rarely used. Therefore, we propose to bring some light on this topic by exploring anthropometric data in a context of high acute malnutrition prevalence like Somalia.

For this purpose we compare the nutrition outcomes yielded by absolute MUAC and MUACAZ with those obtained using WHZ in different populations of Somalia over the 2007-2016 period. We analyse also the effect of factors, namely sex, age and stunting status, expected to contribute to the discrepancy observed between malnutrition rates derived from the three indicators.

\section{Methods}

The data used for this study were obtained from 19 surveys undertaken by FSNAU and partners working in Somalia, in a biannual basis, in the 2007-2016 time period.

There are three main livelihoods in the country: the pastoralists; the agro-pastoralists; and the riverines, that are mainly agrarian [2]. Because of the conflict undergoing in the country, FSNAU also collects data on Internally Displaced Persons (IDPs) which represent a significant proportion of the Somali population. Surveys of IDP camps were included, and these were coded as a further livelihood category although it doesn't constitute a livelihood sensu stricto.

A two-stage cluster sampling with probability proportional to size design was employed where sample sizes for the surveys (number of households and number of children) were calculated using Standardized Methodology for Survey in Relief and Transition (SMART) methods. Previous estimations of global acute malnutrition measured by $\mathrm{WHZ}$ and crude mortality rates for the surveyed areas were used for the calculations. An additional $10 \%$ was added to the sample size to allow for dropout or refusal to participate.

The data were cleaned by deleting the records of individual children with any of the following criteria: age $<6$ months, age $>59$ months, and age, sex, weight, height, oedema or MUAC not recorded. Cases of oedematous malnutrition were excluded from analysis because the WHZ indicator is not valid as proxy of acute malnutrition in such cases $(n=650,0.25 \%$ of the total sample). Weight-for-height and MUAC-forage Z-scores were calculated using WHO Anthro (version 3.2.2, January 2011) and macros using WHO 2006 growth standards. Extreme biologically implausible values were excluded based on WHO standards with recommended flag limits of WHZ and MUAC Z-scores - 5, + 5 [16].

We defined global acute malnutrition (GAM) and severe acute malnutrition (SAM) using the indexes and cut offs described in Table 1 below.

We calculated the prevalence of GAM according to each of the three indicators in a sample pooling the data of the nineteen surveys, stratified by livelihood system, and then, for each of the surveys separately.

For comparing the diagnosis of acute malnutrition using WHZ and absolute MUAC, we defined $\mathrm{WHZ}_{-2}$ only or $\mathrm{WHZ}_{-3}$ only when the child had WHZ below -2 or WHZ below -3 but MUAC over $125 \mathrm{~mm}$ or $115 \mathrm{~mm}$ respectively, and $M U A C_{125}$ only or $M U A C_{115}$ only when MUAC was below 125 or below 115 but WHZ was equal or greater than -2 , or above -3 , respectively. When comparing WHZ and MUACAZ the $\mathrm{WHZ}_{-2}$ only or the $W_{-3}$ only refer to children identified as GAM or SAM by WHZ but not by MUACAZ, and the $M U A C A Z_{-2}$ only and the $M U A C A Z_{-3}$ only to children with MUACAZ below -2 or -3 but above these same values for WHZ.

We performed multivariate logistic regressions on GAM and SAM prevalence defined by WHZ, absolute MUAC and MUACAZ. The independent variables used were sex (male vs female), young age (age $<24$ months vs. age $\geq 24$ months), and stunting (height for age $Z$ score $<-2$ vs height for age $Z$ score $>-2$ ), as they are the hypothetical contributors to the discrepant malnutrition diagnosis between WHZ and absolute MUAC [8]. We tested the association of these independent variables with either $\mathrm{WHZ}_{-2}, \mathrm{MUAC}_{125}$ or $\mathrm{MUACAZ}_{-2}$ for GAM and $\mathrm{WHZ}_{-3}, \mathrm{MUAC}_{115}$ or MUACAZ $\mathrm{MU}_{-3}$ for SAM including all covariates (i.e. sex, young age and stunting) in the overall sample and then in each of the livelihood samples separately, to control for potential confounding and mediating effects.

Stata 14 (StataCorp, College Station, Texas, USA) was used for statistical analysis. 
Table 1 Common anthropometric measurements and indices for children under 5 years of age

\begin{tabular}{|c|c|c|c|}
\hline Index/measurement & Nutritional condition & Indicator & (Acronym) \\
\hline \multirow[t]{2}{*}{ Weight-for-height/length Z score } & Global acute malnutrition & $\mathrm{WHZ}<-2$ & $(\mathrm{WHZ}-2)$ \\
\hline & Severe acute malnutrition & $\mathrm{WHZ}<-3$ & $(\mathrm{WHZ}-3)$ \\
\hline \multirow[t]{2}{*}{ MUAC } & Global acute malnutrition & MUAC $<125 \mathrm{~mm}$ & $\left(\mathrm{MUAC}_{125}\right)$ \\
\hline & Severe acute malnutrition & MUAC $<115 \mathrm{~mm}$ & $\left(\mathrm{MUAC}_{115)}\right.$ \\
\hline \multirow[t]{2}{*}{ MUAC-for-age Z score } & Global acute malnutrition & MUACAZ <-2 & $\left(M \cup A C A Z_{-2}\right)$ \\
\hline & Severe acute malnutrition & MUACAZ $<-3$ & $\left(M \cup A C A Z_{-3)}\right.$ \\
\hline
\end{tabular}

\section{Results}

A total of 255,623 measurements of children aged 6 to 59 months from 19 surveys were examined. There was a nearly equal proportion of girls and boys ( $49 \%$ girls), and the mean age of the children was $31.6( \pm 0.03)$ months, with a distribution of $66 \%$ children with 24 months or older and 33\% children between 6 months and 24 months of age. By livelihood system, 16.5\%, 35.2\% and $10.4 \%$ of children were from areas of agro-pastoral, pastoral and riverine livelihoods, respectively, while 38\% lived in IDPs camps.

\section{Population estimates of acute malnutrition}

In Table 2 we present the prevalence of acute malnutrition (GAM and SAM) as measured by any of the three indicators for the total population, and in each of the livelihoods in Somalia, using the pooled sample of the 2007-2016 surveys.

The prevalence of global acute malnutrition in the total population was very close when calculated by WHZ $_{-2}$ and by MUACAZ -2 (16\% vs. $\left.15.8 \%\right)$, and half of it when calculated by $\mathrm{MUAC}_{125}$ (7.8\%). In pastoralists, the difference was even greater, as the prevalence of GAM by MUAC $_{125}$ was less than half the GAM prevalence yielded by the other two indicators. The SAM estimates showed similar patterns to GAM (Table 2).

The discrepancies between the estimations of GAM by each of the three indicators were sustained throughout the
19 surveys (Fig. 1). In all the surveys the prevalence of GAM was above $12 \%$ when using WHZ $_{-2}$ orMUACAZ $_{-2}$ and much lower when (below $10 \%$ in most of the surveys) using MUAC $_{125}$.

\section{Acute malnutrition screening}

At the individual level, the children identified as acutely malnourished by either three of the indicators were not the same. We compared the individual diagnosis between WHZ and the two MUAC measurements alternatively. In Fig. 2 the pie charts represent the proportion of acutely malnourished children diagnosed by either WHZ and/or absolute MUAC and the pie charts in Fig. 3 represent the proportions of acutely malnourished children that were diagnosed as suchby WHZ and/or MUACAZ.

Figures 2 and 3 show that the overlap in the GAM diagnosis by $\mathrm{WHZ}_{-2}$ and $\mathrm{MUACAZ}_{-2}$ (children identified as GAM by the two indicators simultaneously) was higher than the overlap between $\mathrm{WHZ}_{-2}$ and $\mathrm{MUAC}_{125}$ (28.3 to $18.1 \%$ ). When the analysis was stratified by livelihood the highest overlap between $\mathrm{WHZ}_{-2}$ and $\mathrm{MUAC}_{125}$ was found among agro-pastoralists (20.5\%), and the lowest among pastoralists (14.4\%) (Fig. 2).

The differences for SAM were more pronounced, and the degree of overlap was significantly lower than for GAM, being higher for children identified by $\mathrm{WHZ}_{-3}$ and $\mathrm{MUACAZ}_{-3}(13.0 \%)$ than by $\mathrm{WHZ}_{-3}$ and $\mathrm{MUAC}_{115}$

Table 2 Prevalence of acute malnutrition, defined by $\mathrm{WHZ}^{\mathrm{a}}$, absolute MUAC ${ }^{\mathrm{b}}$ and MUACAZ by four Somalian livelihoods

\begin{tabular}{|c|c|c|c|c|c|c|c|c|c|c|c|c|c|}
\hline \multirow[b]{3}{*}{ Livelihood } & \multirow{3}{*}{$\begin{array}{l}\text { Total subjects } \\
{ }_{n}\end{array}$} & \multicolumn{4}{|c|}{ WHZ } & \multicolumn{4}{|c|}{ MUAC } & \multicolumn{4}{|c|}{ MUACAZ } \\
\hline & & \multicolumn{2}{|c|}{$\mathrm{WHZ}_{-2}$} & \multicolumn{2}{|c|}{$\mathrm{WHZ}_{-3}$} & \multicolumn{2}{|c|}{$\mathrm{MUAC}_{125}$} & \multicolumn{2}{|c|}{ MUAC $_{115}$} & \multicolumn{2}{|c|}{$\mathrm{MUACAZ}_{-2}$} & \multicolumn{2}{|c|}{$\mathrm{MUACAZ}_{-3}$} \\
\hline & & $\%$ & $95 \% \mathrm{Cl}$ & $\%$ & $95 \% \mathrm{Cl}$ & $\%$ & $95 \% \mathrm{Cl}$ & $\%$ & $95 \% \mathrm{Cl}$ & $\%$ & $95 \% \mathrm{Cl}$ & $\%$ & $95 \% \mathrm{Cl}$ \\
\hline Agro-pastoralist & 42,132 & 16.3 & $(16.0-16.7)$ & 3.9 & $(3.8-4.1)$ & 9.6 & $(9.4-9.9)$ & 1.8 & $(1.6-1.9)$ & 19.3 & $(18.9-19.7)$ & 4.2 & $(4.0-4.4)$ \\
\hline Pastoralist & 89,876 & 15.5 & $(15.2-15.7)$ & 3.2 & $(3.1-3.4)$ & 5.7 & $(5.5-5.8)$ & 1.0 & $(0.9-1.1)$ & 13.3 & $(13.1-13.6)$ & 2.2 & $(2.1-2.3)$ \\
\hline Riverine & 26,603 & 16.0 & $(15.6-16.5)$ & 3.9 & $(3.6-4.1)$ & 8.9 & $(8.6-9.3)$ & 1.7 & $(1.6-1.9)$ & 18.2 & $(17.7-18.7)$ & 3.5 & $(3.3-3.8)$ \\
\hline IDPs & 97,012 & 16.5 & $(16.3-16.7)$ & 3.9 & $(3.8-4.0)$ & 8.7 & $(8.5-8.9)$ & 1.9 & $(1.8-2.0)$ & 15.9 & $(15.7-16.1)$ & 3.0 & $(2.9-3.1)$ \\
\hline Total & 255,623 & 16.1 & $(15.9-16.2)$ & 3.7 & $(3.6-3.8)$ & 7.8 & $(7.7-7.9)$ & 1.5 & $(1.4-1.6)$ & 15.8 & $(15.7-15.9)$ & 3.0 & $(2.9-3.1)$ \\
\hline
\end{tabular}

${ }^{a}$ GAM by $W H Z$ defined as $\mathrm{WHZ}<-2$ from the reference population $\left(\mathrm{WHZ}_{-2}\right.$ ) and SAM by $\mathrm{WHZ}$ defined as $\mathrm{WHZ}<-3$ compared to the reference population (WHZ $\left.{ }_{-3}\right)$ ${ }^{\text {b } G A M ~ b y ~ M U A C ~ d e f i n e d ~ a s ~ M U A C ~}<125 \mathrm{~mm}\left(\right.$ MUAC $\left._{125}\right)$ and SAM by MUAC defined as MUAC $<115 \mathrm{~mm}\left(\mathrm{MUAC}_{115}\right)$

'GAM by MUACAZ defined as MUACZ $<-2$ from the reference population (MUAC -2 ) and SAM by MUACAZ defined as MUACAZ $<-3$ compared to the reference population (MUACAZ -3$)$ 


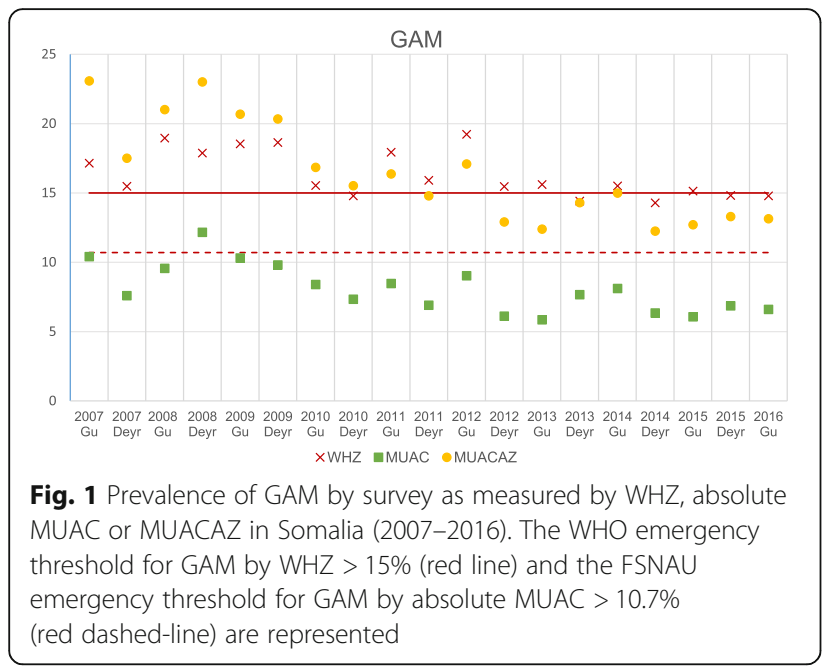

(9.6\%) for the overall population. The largest discrepancy was found for SAM between WHZ and absolute MUAC in pastoralists children.

Out of the 12,170 children diagnosed as SAM by either WHZ or absolute MUAC, only 2799 children would have been identified if $M_{115}$ was the sole criteria to be used, and 8276 if the children were to be diagnosed by $\mathrm{WHZ}_{-3}$ alone. This difference was more marked among the pastoralists population (see Additional file 1: Table S1).

When comparing the SAM diagnosis by WHZ and by MUACAZ the differences were less pronounced, as out of the 15,052 children diagnosed as SAM by either of these two indicators, 7375 children, would have been diagnosed as severely acutely malnourished if $\mathrm{WHZ}_{-3}$ was the sole indicator used, and 5720 if they were to be diagnosed by $\mathrm{MUACAZ}_{-3}$ alone. The difference again was more pronounced among pastoralists (see Additional file 1: Table S2).

\section{Factors associated with acute malnutrition}

In Table 3 we show the results of the multivariate regression on acute malnutrition (GAM and SAM outcomes) as measured by either three of the indicators.

Female children were less likely than boys to be diagnosed as acutely malnourished by WHZ or MUACAZ, whereas they were identified as such more than boys (around $40 \%$ more) with absolute MUAC. On the other hand, stunted children were identified as acutely malnourished about three times more than non-stunted children when MUACAZ and absolute MUAC were used, compared to 1.3 times, at most, when WHZ was the indicator utilized. Among pastoralists children the impact of stunting on the acute malnutrition outcomes was higher than in the other livelihood groups.

Regarding age, there was a change in the direction of the association when the outcome was GAM or SAM, as older children were more likely to be diagnosed as GAM, and less likely to be diagnosed as SAM when measured by WHZ or by MUACAZ in the overall population. Among agropastoralists and pastoralists populations, the children

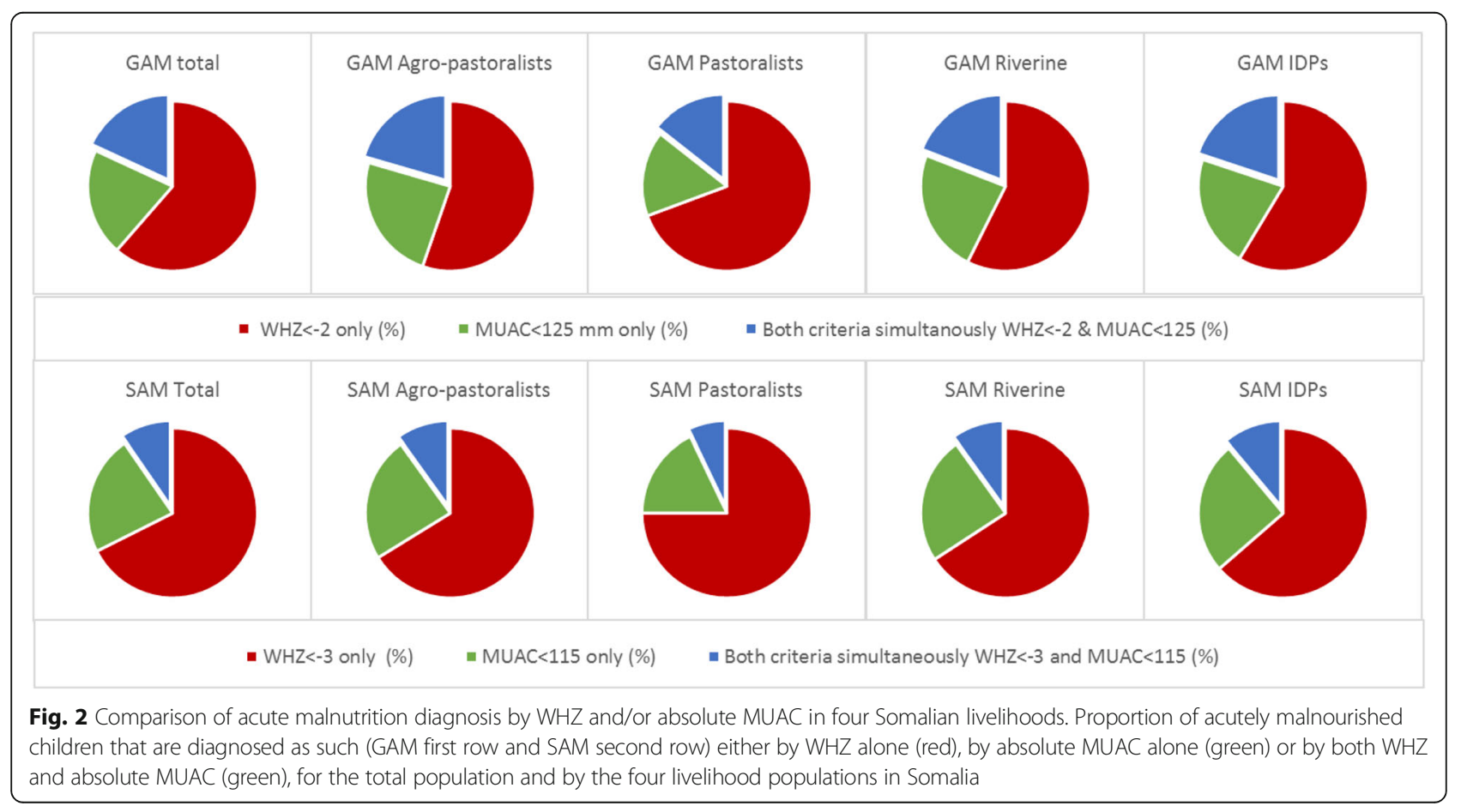




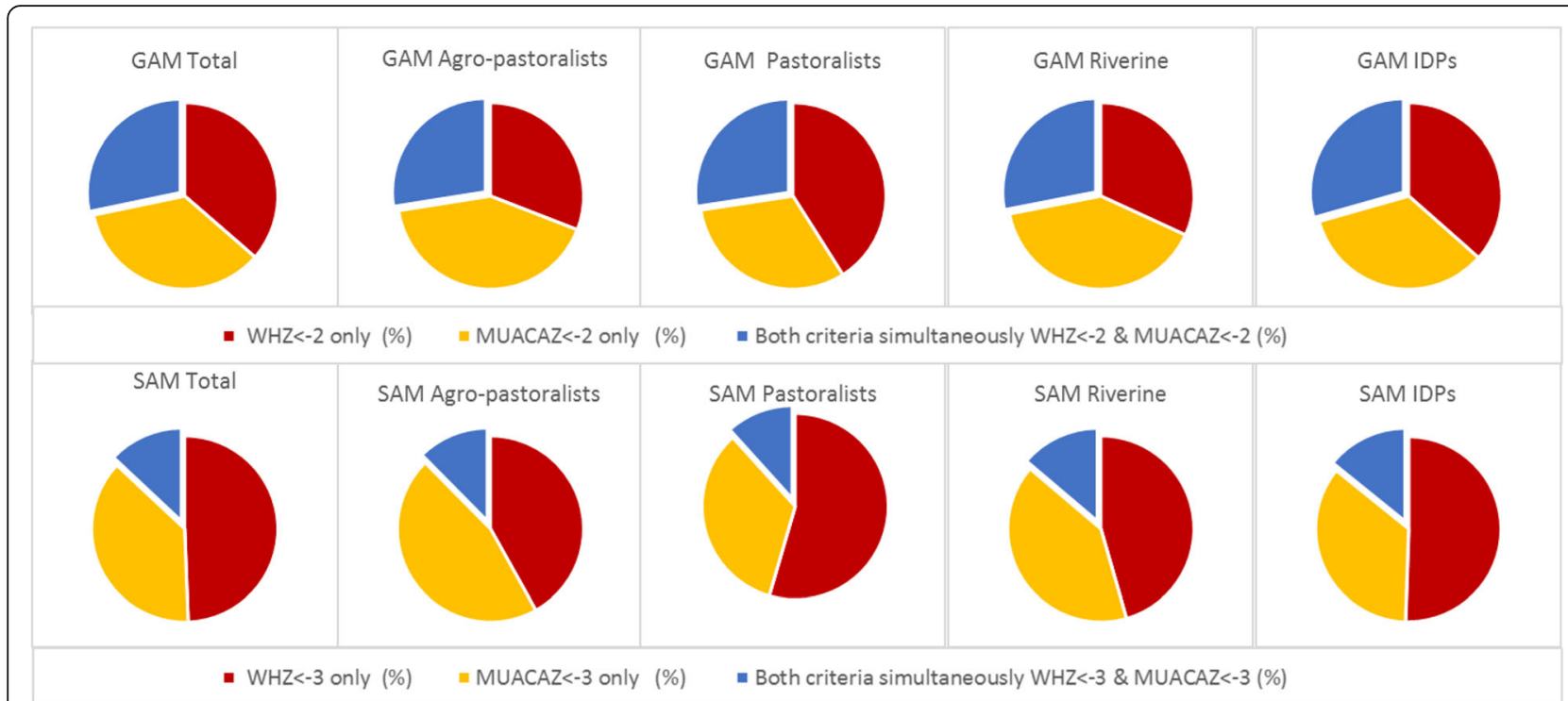

Fig. 3 Comparison of acute malnutrition diagnosis by WHZ and/or MUACAZ in four Somalian livelihoods. Proportion of acutely malnourished that are diagnosed as such (GAM first row and SAM second row) either by WHZ alone (red), by MUACAZ alone (yellow) or by both WHZ and MUACAZ simultaneously (blue) for the overall population and by the four livelihood populations in Somalia

older than 2 years were also more likely to be diagnosed as SAM when using MUACAZ but not when using WHZ (see Additional file 1: Tables S3 and S4), for model results according to livelihood). On the contrary, children older than two years were up to $81 \%$ and $86 \%$ less likely to be diagnosed as GAM or SAM respectively when absolute MUAC was the measure used to diagnose.

\section{Discussion}

We aimed at comparing acute malnutrition estimates as measured by WHZ, absolute MUAC and MUACAZ. A total of 255,623 children aged 6 to 59 months from 19 surveys were included in the analysis. We found an overall GAM prevalence of $16.1 \%$ measured by $\mathrm{WHZ}_{-2}, 7.8 \%$ by MUAC $_{115}$ and $15.8 \%$ by MUACAZ ${ }_{-2}$. Similar results were obtained by livelihood system except for the prevalence of GAM in agro-pastoralists and riverine children that was higher using $\mathrm{MUACAZ}_{-2}$ as compared to $\mathrm{WHZ}_{-2}$, and in pastoralists where the prevalence calculated by $M U_{125}$ was a third of the prevalence by either $\mathrm{WHZ}_{-2}$ or MUA$\mathrm{CAZ}_{-2}$. However, the children identified by either indicator were not the same, being the overlap around $28 \%$ between $\mathrm{WHZ}_{-2}$ and $\mathrm{MUACAZ}_{-2}$ diagnosis, and $18 \%$ between $\mathrm{WHZ}_{-2}$ and $\mathrm{MUAC}_{125}$.

In 1995 the WHO proposed a set of thresholds to classify the severity of malnutrition in emergency situations according to the prevalence of GAM, measured by $\mathrm{WHZ}_{-2}$, that are still widely used ( $<5 \%$ Acceptable, $5-10 \%$ Poor, $10-15 \%$ Serious, $>15 \%$ Critical) [12]. Up to date there are no thresholds to be used at global level when absolute MUAC is used for nutrition surveillance, and using the same thresholds designed for WHZ is problematic. For the majority of the surveys analysed, the classification of the nutrition situation according to WHO thresholds would have been "Critical" when measured by WHZ, and "Poor" when measured by absolute MUAC.

In response to this limitation, FSNAU developed absolute MUAC emergency thresholds adapted to the Somalia context and based on the calculation of the quintiles of the distribution of absolute MUAC. According to those thresholds, the situation is considered "Critical" if the prevalence of GAM as measured by $\mathrm{MUAC}_{125}$ is above $10.7 \%$ [2]. However, in eighteen out of the nineteen surveys analysed the situation would nevertheless have been described as "Critical" by WHO thresholds when using WHZ $_{-2}$ and only "Serious" by FSNAU threshold using $\mathrm{MUAC}_{125}$, thus triggering different responses.

According to our results if the GAM is measured by MUACAZ $_{-2}$ the population estimates are similar to those obtained by $\mathrm{WHZ}_{-2}$, and so they are the resulting classifications according to WHO thresholds in sixteen out of the nineteen surveys analysed.

One of the main arguments for not using MUACAZ it is the age dependency of the indicator, as it is reported that in certain contexts with a high burden of acute malnutrition, age is difficult to ascertain accurately [9]. This may not be the case in Somalia as the field teams have extensive experience in collecting this information due to the high frequency and long history of surveys being conducted in the country. In the sample analyzed the proportion of children 6-23 months old compared to the 24-59 months age group followed the 1:2 expected 


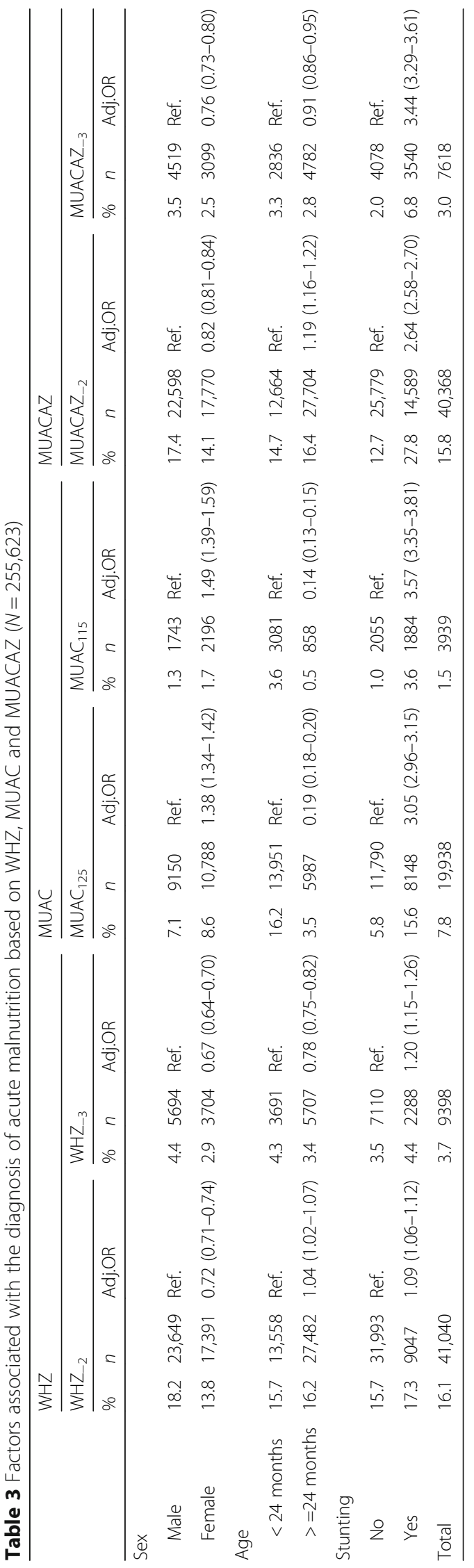


proportion ratio. Moreover, in other emergency nutrition surveillance systems data is collected on both weight-forage and absolute MUAC, suggesting that the age collection is feasible to collect with accuracy [3]. There is also evidence from Bangladesh showing that MUAC-for-age provides comparable information to weight-for-height in the context of nutritional surveillance of populations, and especially for assessing seasonal changes [15].

We recommend to revise the age dependency limitation in view of the importance of bringing age in the estimation of acute malnutrition when using the MUAC measurement. As other authors have already suggested, it could also be possible to construct tally sheets for MUAC divided by age groups in order to minimise the constraints regarding age ascertainment and its accuracy [17].

In relation to the individual screening of acutely malnourished children the results obtained by either indicator did not converge. The discrepancy observed between absolute MUAC and by WHZ in children screening had already been studied $[17,18]$ and taken into account in WHO-UNICEF guidelines [19, 20], but seldom is published on the comparison of children diagnosed as acutely malnourished according to MUACAZ or to WHZ.

The results obtained by Grellety and Golden in their recent review of 47 countries [7] are consistent with our findings regarding the WHZ /MUAC discrepancy in Somalia overall, and the differences we found according to livelihood system were similar to those found by these authors in Ethiopia. Previous studies conducted in Ethiopia found similar results and argued that the prevalence of acute malnutrition by WHZ was overestimated due to differences in body shape such as relatively longer legs that contribute to the total height [21]. However, recent studies refute that this factor alone can explain the discrepancies observed, concluding that they may be due to the combination of different anthropometric characteristics that are expressed differently across populations $[8,11]$.

Moreover, our results disagree with the hypothesis of pastoralist populations showing systematic higher estimates for acute malnutrition as compared to other populations in the same context [22] as they show that the GAM prevalence (according to any of the three measurements) among the pastoralist children was lower than in any of the other livelihood populations studied.

Finally, our results show that age, sex and stunting status of the children also play a role in their likelihood of being diagnosed as acutely malnourished depending on the indicator to be used. Female, younger, and stunted children were more likely to be identified as acutely malnourished if the indicator to be used was absolute MUAC, whereas boys older than two years and stunted were more likely to be diagnosed as GAM by WHZ. Our results confirm those of other studies conducted in countries as Philippines, Chad, South Sudan and Bangladesh [8], Kenya [23] or
South Sudan [7], and some authors have offered the explanation that MUAC is mainly a measure of muscle mass, and that muscle mass is reduced in stunted children $[24,25]$. Interestingly, we found that older boys were more likely to be identified as experiencing GAM by MUACAZ (similar to WHZ results), but also that MUACAZ was the indicator to be more strongly associated with stunting. Consistently, a study in Kenya reported that upper arm muscle area Z-score, an age and gender adjusted measure of absolute lean body mass, explained most of the variability in the progression of stunting among school-aged children over a two year-period [26], suggesting that age adjusted measurements may be more appropriate in the interpretation of weight-for-height and MUAC measurements discrepancies in relation to stunting.

\section{Strengths and limitations}

The sample characteristics in terms of size and number of surveys allowed for a high precision in the analysis, and for the stratification by livelihood system. The so called IDPs livelihood was over-represented in the overall population as recent data indicated that the proportion of IDPs in Somalia population was around 14\% [27], thus the importance of the stratified analysis.

The data were collected in field conditions which may have an impact on the accuracy of measurements, although the FSNAU survey enumerators long time experience and routinely training may have minimized this limitation.

Another limitation of our study is that the analysis was performed on a single country and results cannot be generalizable for other countries.

\section{Conclusions}

The estimates of acute malnutrition by MUACAZ are convergent with the estimations reported by WHZ, as opposed to the high discrepancies found in relation to the absolute MUAC estimates, in our study population. We recommend further research on the MUACAZ indicator in the estimations of GAM in other contexts in which WHZ cannot be collected, as well as the reporting of this indicator in nutrition surveillance reports in Somalia whenever possible.

We encourage the inclusion of MUACAZ in the array of outcomes to be calculated by the available software for nutrition surveillance estimates like the Emergency Nutrition Assessment (ENA) software.

In relation to screening for program admission, we recommend adherence to the current guidelines of using both absolute MUAC and weight-for-height for the screening of severely and moderately malnourished children, and promote research on the role that MUACAZ or alternative indicators can play in this type of surveillance. 


\section{Additional file}

Additional file 1: Table S1. Comparison of acute malnutrition diagnosis by WHZ and/or absolute MUAC in four Somalian livelihoods. Proportion of children with acute malnutrition (GAM or SAM) as defined by $\mathrm{WHZ}$ and/or absolute MUAC (see Methods section for definitions) that are diagnosed as malnourished by WHZ only, by absolute MUAC only, or by both criteria simultaneously. Table S2. Comparison of acute malnutrition diagnosis by WHZ and/or MUACAZ in four Somalian livelihoods.

Proportion of children with acute malnutrition (GAM or SAM) as defined by $\mathrm{WHZ}$ and/or absolute MUACAZ (see Methods section for definitions) that are diagnosed as malnourished by WHZ only, by MUACAZ only, or by both criteria simultaneously. Table S3. Factors associated with GAM diagnosis based on WHZ, absolute MUAC and MUACAZ by livelihood. Results of the multivariate regression on GAM as measured by either three of the indicators (WHZ, MUAC and MUACAZ) conducted in each of the four livelihood populations. Table S4. Factors associated with SAM diagnosis bases on WHZ, MUAC and MUACAZ by livelihood. Results of the multivariate regression on SAM as measured by either three of the indicators (WHZ, MUAC and MUACAZ) conducted in each of the four livelihood populations. (PDF $211 \mathrm{~kb}$ )

\section{Abbreviations}

ENA: Emergency Nutrition Assessment; FSNAU: Food Security and Nutrition Analysis Unit; GAM: Global Acute Malnutrition; IDPs: Internally Displaced Persons; MUAC: Mid-Upper Arm Circumference; MUACAZ: Mid-Upper Arm Circumference Z-score); SAM: Severe Acute Malnutrition;

SMART: Standardized Monitoring and Assessment of Relief and Transitions; UNICEF: United Nations International Children's; WHO: World Health Organization; WHZ: Weight-for-Height Z-score

\section{Acknowledgements}

Not applicable.

\section{Funding}

Not applicable.

\section{Availability of data and materials}

The data that support the findings of this study are available from FSNAU but restrictions apply to the availability of these data, which were used under license for the current study, and so are not publicly available. Data are however available from the authors upon reasonable request and with permission of FSNAU.

\section{Authors' contributions}

EC \& RCM were involved in all stages from the conception and design, data acquisition, analysis and interpretation. FDM was involved in the concept and design of the study. FK contributed to the study design, the data analysis and interpretation. DM \& AY were responsible for conducting the surveys and managing the data. All authors have critically reviewed and approved the final version of the article.

\section{Ethics approval and consent to participate}

FSNAU requested the study, provided the data, and contributed to all steps resulting in this publication.

Ethical approval for data collection was provided through permission by the Ministry of Health Somalia, Transitional Federal Government of Somalia Republic, Ref: MOH/WC/XA/146./07, dated 02/02/07. Owing to the high illiteracy rate of the population, informed verbal consent was sought from all participating households and individuals.

\section{Consent for publication}

Not applicable.

\section{Competing interests}

The authors declare that they have no competing interests.

\section{Publisher's Note}

Springer Nature remains neutral with regard to jurisdictional claims in published maps and institutional affiliations.

\section{Author details}

${ }^{1}$ European Commission, Joint Research Centre, Via E. Fermi, 2749, 21027, Ispra, Varese, Italy. ${ }^{2}$ Food Security and Nutrition Analysis Unit - Somalia, United Nations Food and Agriculture Organization, P.O. Box 30470-00100, Ngecha Road, Nairobi, Kenya.

Received: 21 June 2017 Accepted: 5 February 2018 Published online: 21 February 2018

\section{References}

1. FAO Somalia Nutrition Country Profile. [Internet]. [cited 2017 Mar 15]. Available from: http://www.fao.org/ag/agn/nutrition/som_en.stm

2. Somalia Nutrition Analysis: Post Gu 2016 Technical Series Report No. VII 70 December 21, 2016 [Internet]. Available from: http://fsnau.org/products/ technical-series

3. Bilukha O, Prudhon C, Moloney G, Hailey P, Doledec D. Measuring anthropometric indicators through nutrition surveillance in humanitarian settings: options, issues, and ways forward. Food Nutr Bull. 2012 Jun;33(2):169-76.

4. Tuffrey $V$, Hall A. Methods of nutrition surveillance in low-income countries. Emerg Themes Epidemiol [Internet]. 2016 Mar 18 [cited 2017 Jan 25];13. Available from: http://www.ncbi.n/m.nih.gov/pmc/articles/ PMC4797352/

5. Frison S, Kerac M, Checchi F, Prudhon C. Anthropometric indices and measures to assess change in the nutritional status of a population: a systematic literature review. BMC Nutr. 2016;2:76.

6. The arm circumference as a public health index of protein-calorie malnutrition of early childhood. J Trop Pediatr 1967. 1969 Dec;15(4):177-260.

7. Grellety E, Krause LK, Shams Eldin M, Porten K, Isanaka S. Comparison of weight-for-height and mid-upper arm circumference (MUAC) in a therapeutic feeding programme in South Sudan: is MUAC alone a sufficient criterion for admission of children at high risk of mortality? Public Health Nutr. 2015 Oct;18(14):2575-81.

8. Roberfroid D, Huybregts L, Lachat C, Vrijens F, Kolsteren P, Guesdon B. Inconsistent diagnosis of acute malnutrition by weight-for-height and mid-upper arm circumference: contributors in 16 cross-sectional surveys from South Sudan, the Philippines, Chad, and Bangladesh. Nutr J. 2015;14:86.

9. Myatt M, Khara T, Collins S. A review of methods to detect cases of severely malnourished children in the community for their admission into community-based therapeutic care programs, 2006. Food Nutr Bull. 27(no.3 (supplement));S7-S23.

10. Isanaka S, Guesdon B, Labar AS, Hanson K, Langendorf C, Grais RF. Comparison of clinical characteristics and treatment outcomes of children selected for treatment of severe acute malnutrition using mid upper arm circumference and/or weight-for-height Z-score. PLoS One. 2015;10(9):e0137606.

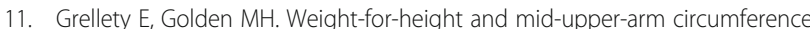
should be used independently to diagnose acute malnutrition: policy implications. BMC Nutr. 2016:2:10.

12. WHO Expert Committee. WHO | Physical status: the use and interpretation of anthropometry. Technical Report Series No. 854. [Internet]. 1995 [cited 2017 Jan 31]. Available from: http://www.who.int/childgrowth/publications/ physical_status/en/

13. de Onis M, Yip R, Mei Z. The development of MUAC-for-age reference data recommended by a WHO expert committee. Bull World Health Organ. 1997:75(1):11-8

14. Hall G, Chowdhury S, Bloem M. Use of mid-upper-arm circumference Z scores in nutritional assessment. Lancet Lond Engl. 1993;341(8858):1481.

15. Brown KH, Black RE, Becker S. Seasonal changes in nutritional status and the prevalence of malnutrition in a longitudinal study of young children in rural Bangladesh. Am J Clin Nutr. 1982;36(2):303-13.

16. World Health Organization. The WHO Child Growth Standards [Internet]. WHO. [cited 2017 Jan 26]. Available from: http://www.who.int/childgrowth/en/

17. Fiorentino $M$, Sophonneary $P$, Laillou $A$, Whitney $S$, de Groot $R$, Perignon $M$, et al. Current MUAC Cut-Offs to Screen for Acute Malnutrition Need to Be 
Adapted to Gender and Age: The Example of Cambodia. PLoS ONE. 2016; 11(2):e0146442. https://doi.org/10.1371/journal.pone.0146442

18. Fernández MÁL, Delchevalerie P, van HM. Accuracy of MUAC in the detection of severe wasting with the new WHO growth standards. Pediatrics. 2010;126(1):e195-201.

19. World Health Organization. Assessment and surveillance of nutritional status in emergencies 2010; 10 May 2010. [Internet]. WHO. [cited 2017 Jan 25]. Available from: http://www.who.int/hac/techguidance/training/ Day\%204\%20nutrition\%20assessment.pdf

20. WHO |WHO child growth standards and the identification of severe acute malnutrition in infants and children [Internet]. WHO. [cited 2017 Jan 25]. Available from: http://www.who.int/nutrition/publications/ severemalnutrition/9789241598163/en/

21. Myatt M, Duffield A, Seal A, Pasteur F. The effect of body shape on weightfor-height and mid-upper arm circumference based case definitions of acute malnutrition in Ethiopian children. Ann Hum Biol. 2009;36(1):5-20.

22. Mason JB, White JM, Heron L, Carter J, Wilkinson C, Spiegel P. Child acute malnutrition and mortality in populations affected by displacement in the horn of Africa, 1997-2009. Int J Environ Res Public Health. 2012;9(3):791-806.

23. Berkley J, Mwangi I, Griffiths K, Ahmed I, Mithwani S, English M, et al. Assessment of severe malnutrition among hospitalized children in rural Kenya: comparison of weight for height and mid upper arm circumference. JAMA. 2005;294(5):591-7.

24. Frisancho AR, Garn SM, McCreery LD. Relationship of skinfolds and muscle size to growth of children. I. Costa Rica. Am J Phys Anthropol. 1971;35(1):85-90.

25. Soares-Wynter SY, Walker SP. Resting metabolic rate and body composition in stunted and nonstunted children. Am J Clin Nutr. 1996;64(2):137-41.

26. Friedman JF, Phillips-Howard PA, Mirel LB, Terlouw DJ, Okello N, Vulule JM, et al. Progression of stunting and its predictors among school-aged children in western Kenya. Eur J Clin Nutr. 2005;59(8):914-22.

27. Refugees in the Horn of Africa: Somali Displacement Crisis - Somalia [Internet]. [cited 2017 Jun 19]. Available from: http://data.unhcr.org/horn-ofafrica/country.php?id=19

\section{Submit your next manuscript to BioMed Central and we will help you at every step:}

- We accept pre-submission inquiries

- Our selector tool helps you to find the most relevant journal

- We provide round the clock customer support

- Convenient online submission

- Thorough peer review

- Inclusion in PubMed and all major indexing services

- Maximum visibility for your research

Submit your manuscript at www.biomedcentral.com/submit 